\title{
Traditional Chinese Medicine in orthopaedics-Problems and future direction
}

\author{
Ping-Chung Leung ${ }^{1,2,3}$ \\ ${ }^{1}$ Department of Orthopaedics \& Traumatology, The Chinese University of Hong Kong, Hong Kong, China; pingcleung@cuhk.edu.hk \\ ${ }^{2}$ Jockey Club Centre for Osteoporosis Care \& Control, The Chinese University of Hong Kong, Hong Kong, China \\ ${ }^{3}$ Centre for Clinical Trials on Chinese Medicine, Institute of Chinese Medicine, The Chinese University of Hong Kong, Hong Kong, \\ China
}

Received 30 October 2013; revised 9 December 2013; accepted 24 December 2013

Copyright (C) 2014 Ping-Chung Leung. This is an open access article distributed under the Creative Commons Attribution License, which permits unrestricted use, distribution, and reproduction in any medium, provided the original work is properly cited. In accordance of the Creative Commons Attribution License all Copyrights (c) 2014 are reserved for SCIRP and the owner of the intellectual property Ping-Chung Leung. All Copyright (C 2014 are guarded by law and by SCIRP as a guardian.

\section{ABSTRACT}

Introduction: Orthopaedic surgery includes varieties of injuries and other pathologies. Treatments are complicated while healing and recovery take time. Rehabilitation needs planning and commitment. In the Chinese Communities, traditional medicine has been popular since hundreds of years ago and has remained so in spite of the current advances in medicine. The purpose of this review is to identify the techniques being used in current situations, the indications and problems. Varieties of Traditional Treatments: 1) Topical applications of herbs are very much welcome after joint sprains, inflammatory conditions and even fractures. 2) On the wide area of physical training, the Chinese Taichi and Qigong are excellent gentle exercises for personal pursues. These exercises emphasize on body-mind interactions and carry strong philosophical insights. 3) Acupuncture has become standard rehabilitation practice in some hospitals and is getting even more popular in pain control and rehabilitation training after nerve injuries. Problems: Topical agents for pain and inflammation are not totally safe because allergic adverse conditions could happen from time to time. The major concern could be the unawareness of non-unions or delayed unions in fracture cases after herbal applications because the traditional healers are using too little modern assessment tools. Physical trainings and acupunctures are on the whole safe, only that indications are not clearly defined. Future Direction: 1) Concerning the topical agents used for pain and swelling, the essential research requirements include demonstration on the topical agents' anti inflammatory, angiogenic and cell regenerative effects. The capability of skin penetration needs to be confirmed and a final clinical trial will further confirm the efficacy. 2) Clinical researches trying to obtain evidence on the use of Tai-chi and Qigong on the neuromuscular recovery are plentiful, while their effects on cardiovascular and respiratory health are likewise encouraging. 3) Acupuncture has already become a standard treatment option for pain control, nausea/vomiting and neuromuscular recoveries.

\section{KEYWORDS}

Orthopedics; Rehabilitation; Traditional Medicine; Topical Agent; Tai-chi; Qigong

\section{INTRODUCTION}

The popular use of Traditional Chinese Medicine for Orthopaedic conditions has stood well against the test of time, not only in rural communities but also in city areas. Topical applications of herbal preparations are particularly welcome in joint sprains, inflammatory conditions and even in bone fractures [1]. At the same time, we encounter skin allergies in response to topical applications, stiff joints and fracture mal-alignments [2]. Opposing views are therefore widely heard: orthopaedic colleagues tend to be very negative while bone setters continue to make energetic claims which are supported by users.

As a matter of fact, Traditional Chinese Medicine has three main offers in orthopedics, viz. topical agents for 
local treatment, systemic herbal formulae for musculoskeletal diseases and regiments of combined practices for rehabilitation training (understood as "manual therapy”) [3-5]. In the current scientific era, experimental platforms are available to prove or disprove the efficacy of the different preparations and methods being used in the three major areas.

\section{THREE MAJOR AREAS OF APPLICATION IN ORTHOPEDICS}

\subsection{Topical Agents}

If topical agents reduce pain and swelling, and promote healing, they must be shown to be able to penetrate the skin surface, then produce anti-inflammatory effects and angiogenic effects, while not causing ill health or allergic reactions.

Indeed experimental reports using respectable research platforms have been appearing [3,6]. The work being done in Hong Kong is presented as follows:

To investigate whether topical agents of herbal origin used in Traditional Chinese Medicine have real biological effects on limb swelling and tissue healing, formation of an innovative formula was achieved through a literature screening of over 200 herbs. The herbs selected have been reported to be anti-inflammatory, promoting circulation and supporting tissue/bone healing. In vitro and in vivo tests were done to investigate the biological effects of the herbs. When used as a topical agent for tissue swelling: 1) The herbs were found to be able to be transported as chemicals through an artificial membrane and porcine skin in a diffusion chamber; 2) when used as a herbal bath, it was found to positively reduce hand and foot swelling. The topical agent was also used for fracture healing: 1) In vitro tests showed positive anti-inflammatory, circulation promotion and tissue healing effects; 2) treated animals showed bigger callus, higher serum bone specific alkaline phosphatase and better mechanical strength; and 3) liquid chromatograph and mass spectrometry testing showed that there was a real transport of chemical markers across the skin. We therefore could conclude that Traditional Chinese Medicine herbal agents used topically for hand swelling and tissue healing do have a demonstrable scientific basis.

\subsection{Systemic Treatment for Musculoskeletal Diseases}

While pharmaceuticals work on single pathological targets and produce quick responses, herbal formulae work on multiple targets to produce slow cumulative effects. Clinical researches to verify the efficacy of herbal formulae need to address the multiple issues of herbal authentication, biological activities involved and finally evidence based clinical trials. One frequently tackled area involves the rheumatological conditions since modern standard treatment might yet find disappointments. A number of anti-inflammatory herbal formulae are available in Chinese Medicine classics and have likewise been tested in different institutions [4,7,8]. The work being done in Hong Kong is presented as follows:

To examine the safety and efficacy of a Traditional Chinese Medicine consisting of Lingzhi and San Miao San, including their antioxidant and immuno-modulating properties in rheumatoid arthritis (RA), a 24-week placebo-controlled study included patients with active disease despite treatment with disease modifying drugs were recruited. 32 patients were randomized to receive Traditional Chinese Medicine (Lingzhi $4 \mathrm{gm}$ and San Miao San 2.4 gm daily) and 33 on placebo. The primary outcome was the number of patients with American College of Rheumatology score (ACR) reaching 20 and the secondary outcomes included changes in pain score, number of swollen and tender joints, ESR, CRP, physician's and patient's global score (VAS), HAQ and cytokine levels, and oxidative stress markers.

Results showed that of the 58 (89.2\%) patients completed 24 weeks study. 5/32 (15.6\%) patients in the Traditional Chinese Medicine group vs 3/33 (9.1\%) patients in the placebo group $(\mathrm{p}<0.05)$ achieved ACR 20. Pain control and swollen joint control increased significantly in the Traditional Chinese Medicine group. Patient's global score improved significantly in both groups. Tender joint counts, ESR, CRP; oxidative stress markers remained unchanged. Plasma CRP and the percentage, absolute counts and the ratio of $\mathrm{CD} 4+/ \mathrm{CD} 8+/ \mathrm{NK} / \mathrm{B}$ lymphocytes were unchanged between groups. CD3, CD4, CD8 lymphocytes counts were unchanged. However, the levels of plasma IL-18, interferon-gamma-induced protein-10 (IP-10), and IL-8 were significantly decreased in the Traditional Chinese Medicine group. In ex vivo experiment, the percentage change of IL-18 was significantly lower but IP-10 was significantly elevated in the Traditional Chinese Medicine group, 13 patients reported 23 episodes (15 in placebo group and 8 in Traditional Chinese Medicine group) of mild adverse effects.

Conclusions were made that Traditional Chinese Medicine was an effective analgesic agent for refractory RA, and was safe and well tolerated. In additional, mild anti-inflammatory effects were seen although immunoregulatory effects but the anti-oxidant effects could not be demonstrated.

\subsection{Rehabilitation Procedures}

Rehabilitation procedures like Tai Chi and Qi Gong have become popular in Europe and America as means to 
maintain healthy aging [9]. In 1999, the National Institutes of Health US, held a consensus conference, endorsing the use of acupuncture as effective means of pain and nausea control. Since then major hospitals in US all established acupuncture services. Acupuncture and manual therapies of course are also useful for the promotion of neurological recovery [10]. With the development of modern imaging techniques like functional MRI, and discoveries of unexpected neurological functional pathways, the course of events of acupuncture is being revealed gradually [11].

In the 1980's there were plenty of studies reporting on the efficacy of electrical stimulation, either with TENS or acupuncture for back pain. Coan [12] reported that patients receiving a minimal of eight acupuncture treatments experienced less pain at a 40-week follow-up than those who dropped out after only one to two treatments. Lehmann, et al. [13] conducted a trial to assess the efficacy of TENS and electro-acupuncture (EAP) in the rehabilitation of chronic back pain patients. Fifty-four patients treated in a 3-week inpatient rehabilitation program were randomly assigned to and accepted treatment with EAP TENS (low intensity transcutaneous nerve stimulation) and TENS dead-battery (placebo). Outcome measures included estimates of pain (on a Visual Analogue Scale) and disability by both physician and patient, physical measures of trunk strength and range of motion of the spine, as well as the patient's perceptions of the relative contribution of the education, exercise training, and the electrical simulation. Analyses of variance were utilized to determine effects of treatment (EAP, TENS, and placebo) across time (admission, discharge, and return) for the outcome measures. There were no significant differences between treatment groups with respect to their overall rehabilitation. All three treatment groups ranked the contribution of the education as being greater than the electrical stimulation. However, the EAP group consistently demonstrated greater improvement on the outcome measures than the other treatment groups. For the Visual Analogue Scale measure of average pain, there was a statistical trend at the return visit, suggesting that the acupuncture group was experiencing less pain.

A recent Cochrane review of 35 trials, evaluating the effectiveness of acupuncture for treatment of non-specific low-back pain and dry-needling for myofascial pain syndrome in the low-back region compared to no treatment, sham therapies, and the addition of acupuncture to other therapies, provided good evidence on the effectiveness of acupuncture for chronic low-back pain [14]. The results suggested that acupuncture is more effective for pain relief and functional improvement compared to no treatment and sham therapies during follow-up. However, acupuncture was not more effective than other conventional and "alternative" treatments when compar- ing its effectiveness with other conventional treatments. The evidence suggested that acupuncture is more useful for pain relief and functional improvement when used in conjunction with other conventional therapies than when used alone. The reviewers concluded that acupuncture may be useful as either a unique therapy for chronic lowback pain or an adjunct therapy to other conventional treatments.

The conclusion at this stage could be as follows: although the effect of acupuncture on pain control is nothing like the use of analgesics that bring immediate, but short-term relief, it has great practical value as an alternative for chronic persistent pain. The mechanism of action of acupuncturing is yet unclear. Recent studies using functional MRI indicated that the functional activities in specific regions of the brain could be mapped out during procedures of acupuncture [15]. With further maturation of the basic research trying to explain this ancient art of healing, acupuncture is expected to become even more popular in time. Acupuncture has been the most popular form of alternative treatment in modern hospitals and clinics, especially among pain teams. For the clinician, it will be to his disadvantage if he does not realize the potential value of acupuncture for his patients suffering from resistant, chronic pain.

\section{PROBLEMS}

Normally orthopaedic surgeons organise their own operations and rehabilitation programs which consist of mainly physiotherapy and occupational therapies. Even in a so called "integrated clinic", surgeons prefer not having traditional clinicians involved. While the traditional practitioners serve their own clients with relatively milder presentation, they would refer them to the surgeons when complications occur. Allergic reactions to topical herbal applications are not uncommon. Antiallergic therapy using anti histamines is usually successful. Acupuncture occasionally cause vascular damages which must be extremely rare. Complications arising from the traditional forms of exercise training are virtually unheard. The controversy of Traditional Chinese Medicine in orthopaedics has resulted from a lack of genuine dialogue between the orthopaedic surgeon and the traditional practitioner. The former is busy with more and more advanced techniques in surgery and therapeutic measures while the latter feel isolated and so deficient of the background to acquire the modern informations and technologies.

\section{FUTURE DIRECTION}

A frank dialogue between the orthopedic surgeon and the traditional practitioner would bring a bright future to patients suffering from bone and joint diseases [16]. The 
immediate result of the dialogue would be a reasonable division of labour. Emergencies and surgeries would follow strict indications and guidelines of modern practice to be executed by modern practitioners. Chronic problems and rehabilitation would need the input of traditional experts. On the prevention side, it would be a great blessing to all if life style considerations could be diligently included, on top of modern public health measures like vaccinations and strict hygiene controls. A system of personal practices which help the individual to avoid falling sick and resisting the attacks of diseases has been a strong classical practice for the traditional healers [9] and needs to be revitalized. Modern research platforms would reveal more and more biological evidences of old practices, and more effective innovations will emerge for the control of inflammation, revascularization and regeneration.

\section{ACKNOWLEDGEMENTS}

This study was supported by grants on the State Key Laboratory of Phytochemistry and Plant Resources in West China (CUHK) from HKSAR and CUHK.

\section{REFERENCES}

[1] Jiang, S.Y. and Ye, G.C. (2005) Authentication of "bone penetrating herbs”. Lishizhen Medicine and Materia Medica Research, 16, 1014-1015.

[2] Lee, T.Y. and Law, T.H. (1991) Bone setters' herbs dermatitis in Hong Kong. Contact Dermatitis, 24, 304. http://dx.doi.org/10.1111/j.1600-0536.1991.tb01730.x

[3] Leung, P.C., Peng, L.H., Zhao, X. and Hung, L.K. (2010) Rehabilitation using topical agent-Myth or reasonable option? Hong Kong Journal of Occupational Therapy, 20, 3-7. http://dx.doi.org/10.1016/S1569-1861(10)70051-6

[4] Li, E.K., Tam, L.S., Wong, C.K., Li, W.C., Lam, C.W.K., Wachtel-Galor, S., Benzie, I.F., Bao, Y.X., Leung, P.C. and Tomlinson, B. (2007) Safety and efficacy of Ganoderma lucidum (Lingzhi) and San Miao San supplementation in patients with rheumatoid arthritis: A doubleblind, randomized, placebo-controlled pilot trial. Arthritis \& Rheumatism, 57, 1143-1150. http://dx.doi.org/10.1002/art.22994
[5] Leung, P.C. (2010) Natural healing in Chinese medicine: Qi Gong and Tai Chi. In: Leung, P.C. and Xue, C.C., Eds., Healthy Aging, Annals of Traditional Chinese Medicine, World Scientific Publisher, Singapore, 221-242.

[6] Leung, P.C., Zhao, X., Law, W.T. and Lau, T.W.D. (2007) Can herbal extracts be used as skin penetrating agent. Oriental Pharmacy and Experimental Medicine, 7, 211216. http://dx.doi.org/10.3742/OPEM.2007.7.3.211

[7] Xu, J.H., Li, C.C. and Huang, Z.Q. (1989) Effect of triptolide on cytokinetics of hela cells. Acta Pharmacological Sinica, 10, 550-553.

[8] Casimiro, L., Barnsley, L., Brosseau, L., Milne. S., Robinson, V.A. Tugwell, P. and Wells, G. (2005) Acupuncture and Electroacupuncture for treatment of Rheumatoid Arthritis. The Cochrane Database of Systematic Reviews, 4, CD003788.

[9] Leung, P.C. (2009) Happy aging. Chinese Journal of Integrative Medicine, 15, 466-469.

[10] National Institutes of Health (NIH) (1997) Acupuncture. NIH Consensus Statement, 15, 1-34. http://consensus.nih.gov/1997/1997acupuncture107html.h $\underline{\mathrm{tm}}$

[11] Cho, Z.H, Wong, E.K. and Fallon, J.H. (2001) Neuroacupuncture-Neuroscience basics. Q-Puncture Inc., Los Angeles.

[12] Coan, R.M., Wong, G. and Coan, P.L. (1981) Acupuncture treatment of neck pain: A randomized controlled study. American Journal of Chinese Medicine, 9, 326-332. http://dx.doi.org/10.1142/S0192415X81000433

[13] Lehmann, T.R., Russell, D.W., Spratt, K.F., Colby, H., Liu, Y.K., Fairchild, M.L. and Christensen, S. (1986) Efficacy of electro-acupuncture and TENS in the rehabilitation of chronic low back pain patients. Pain, 26, 277-290. http://dx.doi.org/10.1016/0304-3959(86)90057-6

[14] Furlan, A.D., Van Tulder, M.W., Cherkin, D.C., Tsukayama, H., Lao, L., Koes, B.W. and Berman, B.M. (2005) Acupuncture and dry-needling for low back pain. The Cochrane Database of Systematic Reviews, 1, CD001351

[15] Yoo, S.S., Teh, E.K., Blinder, R.A. and Jolesz, F.A. (2004) Modulation of cerebellar activities by acupuncture stimulation: Evidence from fMRI study. Neurolmage, 22, 932940. http://dx.doi.org/10.1016/j.neuroimage.2004.02.017

[16] Leung, P.C. (2006) A practical way of research in Chinese medicine. Annals Academy of Medicine, Singapore, 35, 770-772. 